\title{
Research Article \\ Generalized Vector-Valued Sequence Spaces Defined by Modulus Functions
}

\author{
Mahmut Işik

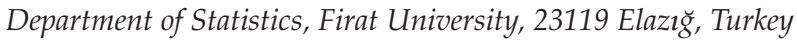 \\ Correspondence should be addressed to Mahmut Işik, misik63@yahoo.com \\ Received 17 June 2010; Accepted 16 December 2010 \\ Academic Editor: Alberto Cabada
}

Copyright (C) 2010 Mahmut Işik. This is an open access article distributed under the Creative Commons Attribution License, which permits unrestricted use, distribution, and reproduction in any medium, provided the original work is properly cited.

We introduce the vector-valued sequence spaces $w_{\infty}\left(\Delta^{m}, F, Q, p, u\right), w_{1}\left(\Delta^{m}, F, Q, p, u\right)$, and $w_{0}\left(\Delta^{m}, F, Q, p, u\right), S_{u}^{q}$ and $S_{0 u^{\prime}}^{q}$ using a sequence of modulus functions and the multiplier sequence $u=\left(u_{k}\right)$ of nonzero complex numbers. We give some relations related to these sequence spaces. It is also shown that if a sequence is strongly $\Delta^{m} u_{q}$-Cesàro summable with respect to the modulus function $f$ then it is $\Delta^{m} u_{q}$-statistically convergent.

\section{Introduction}

Let $w$ be the set of all sequences real or complex numbers and $\ell_{\infty}, c$, and $c_{0}$ be, respectively, the Banach spaces of bounded, convergent, and null sequences $x=\left(x_{k}\right)$ with the usual norm $\|x\|=\sup \left|x_{k}\right|$, where $k \in \mathbb{N}=\{1,2, \ldots\}$, the set of positive integers.

The studies on vector-valued sequence spaces are done by Das and Choudhury [1], Et [2], Et et al. [3], Leonard [4], Rath and Srivastava [5], J. K. Srivastava and B. K. Srivastava [6], Tripathy et al. [7, 8], and many others.

We define

Let $\left(E_{k}, q_{k}\right)$ be a sequence of seminormed spaces such that $E_{k+1} \subset E_{k}$ for each $k \in \mathbb{N}$.

$$
w(E)=\left\{x=\left(x_{k}\right): x_{k} \in E_{k} \text { for each } k \in \mathbb{N}\right\} .
$$

It is easy to verify that $w(E)$ is a linear space under usual coordinatewise operations defined by $x+y=\left(x_{k}+y_{k}\right)$ and $\alpha x=\left(\alpha x_{k}\right)$, where $\alpha \in \mathbb{C}$.

Let $u=\left(u_{k}\right)$ be a sequences of nonzero scalar. Then for a sequence space $E$, the multiplier sequence space $E(u)$, associated with the multiplier sequence $u$, is defined as $E(u)=\left\{\left(x_{k}\right) \in w:\left(u_{k} x_{k}\right) \in E\right\}$. 
The notion of a modulus was introduced by Nakano [9]. We recall that a modulus $f$ is a function from $[0, \infty)$ to $[0, \infty)$ such that

(i) $f(x)=0$ if and only if $x=0$,

(ii) $f(x+y) \leq f(x)+f(y)$ for $x, y \geq 0$,

(iii) $f$ is increasing,

(iv) $f$ is continuous from the right at 0 .

It follows that $f$ must be continuous everywhere on [0, $\infty)$. Maddox [10] and Ruckle [11] used a modulus function to construct some sequence spaces.

After then some sequence spaces defined by a modulus function were introduced and studied by Bilgin [12], Pehlivan and Fisher [13], Waszak [14], Bhardwaj [15], Altın [16], and many others.

The notion of difference sequence spaces was introduced by Kizmaz [17] and it was generalized by Et and Çolak [18]. Let $m$ be a fixed positive integer. Then we write

$$
X\left(\Delta^{m}\right)=\left\{x=\left(x_{k}\right):\left(\Delta^{m} x_{k}\right) \in X\right\}
$$

for $X=\ell_{\infty}, c$ and $c_{0}$, where $m \in \mathbb{N}, \Delta^{m} x=\left(\Delta^{m-1} x_{k}-\Delta^{m-1} x_{k+1}\right), \Delta^{0} x=\left(x_{k}\right)$ and so we have

$$
\Delta^{m} x_{k}=\sum_{v=0}^{m}(-1)^{v}\left(\begin{array}{l}
m \\
v
\end{array}\right) x_{k+v}
$$

\section{Main Results}

In this section, we prove some results involving the sequence spaces $w_{0}\left(\Delta^{m}, F, Q, p, u\right)$, $w_{1}\left(\Delta^{m}, F, Q, p, u\right)$, and $w_{\infty}\left(\Delta^{m}, F, Q, p, u\right)$.

Definition 2.1. Let $\left(E_{k}, q_{k}\right)$ be a sequence of seminormed spaces such that $E_{k+1} \subset E_{k}$ for each $k \in \mathbb{N}, p=\left(p_{k}\right)$ a sequence of strictly positive real numbers, $Q=\left(q_{k}\right)$ a sequence of seminorms, $F=\left(f_{k}\right)$ a sequence of modulus functions, and $u=\left(u_{k}\right)$ any fixed sequence of nonzero complex numbers $u_{k}$. We define the following sequence spaces:

$$
\begin{aligned}
& w_{0}\left(\Delta^{m}, F, Q, p, u\right)=\left\{x=\left(x_{k}\right): x_{k} \in E_{k}: \frac{1}{n} \sum_{k=1}^{n}\left[f_{k}\left(q_{k}\left(u_{k} \Delta^{m} x_{k}\right)\right)\right]^{p_{k}} \longrightarrow 0, \text { as } n \longrightarrow \infty\right\}, \\
& w_{1}\left(\Delta^{m}, F, Q, p, u\right)=\left\{x=\left(x_{k}\right): x_{k} \in E_{k}: \frac{1}{n} \sum_{k=1}^{n}\left[f_{k}\left(q_{k}\left(u_{k} \Delta^{m} x_{k}-\ell\right)\right)\right]^{p_{k}} \longrightarrow 0,\right. \\
&\text { as } \left.n \longrightarrow \infty, \ell \in E_{k}\right\}, \\
& w_{\infty}\left(\Delta^{m}, F, Q, p, u\right)=\left\{x=\left(x_{k}\right): x_{k} \in E_{k}: \sup _{n} \frac{1}{n} \sum_{k=1}^{n}\left[f_{k}\left(q_{k}\left(u_{k} \Delta^{m} x_{k}\right)\right)\right]^{p_{k}}<\infty\right\} .
\end{aligned}
$$


Throughout the paper $Z$ will denote any one of the notation 0,1 or $\infty$.

If $f_{k}=f$ and $q_{k}=q$ for all $k \in \mathbb{N}$, we will write $w_{Z}\left(\Delta^{m}, f, q, p, u\right)$ instead of $w_{Z}\left(\Delta^{m}, F, Q, p, u\right)$.

If $f(x)=x$ and $p_{k}=1$ for all $k \in \mathbb{N}$, we will write $w_{Z}\left(\Delta^{m}, q, u\right)$ instead of $w_{Z}\left(\Delta^{m}, f, q, p, u\right)$.

If $x \in w_{1}\left(\Delta^{m}, f, q, p, u\right)$, we say that $x$ is strongly $\Delta^{m} u_{q}$-Cesàro summable with respect to the modulus function $f$ and we will write $x_{k} \rightarrow \ell\left(w_{1}\left(\Delta^{m}, f, q, p, u\right)\right)$ and $\ell$ will be called $\Delta^{m} u_{q}$-limit of $x$ with respect to the modulus $f$.

The proofs of the following theorems are obtained by using the known standard techniques; therefore, we give them without proofs.

Theorem 2.2. Let the sequence $\left(p_{k}\right)$ be bounded. Then the spaces $w_{Z}\left(\Delta^{m}, F, Q, p, u\right)$ are linear spaces.

Theorem 2.3. Let $f$ be a modulus function and the sequence $\left(p_{k}\right)$ be bounded; then

$$
w_{0}\left(\Delta^{m}, f, q, p, u\right) \subset w_{1}\left(\Delta^{m}, f, q, p, u\right) \subset w_{\infty}\left(\Delta^{m}, f, q, p, u\right)
$$

and the inclusions are strict.

Theorem 2.4. $w_{0}\left(\Delta^{m}, F, Q, p, u\right)$ is a paranormed (need not total paranorm) space with

$$
g_{\Delta}(x)=\sup _{n}\left(\frac{1}{n} \sum_{k=1}^{n}\left[f_{k}\left(q_{k}\left(u_{k} \Delta^{m} x_{k}\right)\right)\right]^{p_{k}}\right)^{1 / M}
$$

where $M=\max \left(1, \sup p_{k}\right)$.

Theorem 2.5. Let $F=\left(f_{k}\right)$ and $G=\left(g_{k}\right)$ be any two sequences of modulus functions. For any bounded sequences $p=\left(p_{k}\right)$ and $t=\left(t_{k}\right)$ of strictly positive real numbers and for any two sequences of seminorms $q=\left(q_{k}\right)$ and $r=\left(r_{k}\right)$, we have

(i) $w_{Z}\left(\Delta^{m}, f, Q, u\right) \subset w_{Z}\left(\Delta^{m}, f \circ g, Q, u\right)$;

(ii) $w_{Z}\left(\Delta^{m}, F, Q, p, u\right) \cap w_{Z}\left(\Delta^{m}, F, R, p, u\right) \subset w_{Z}\left(\Delta^{m}, F, Q+R, p, u\right)$;

(iii) $w_{Z}\left(\Delta^{m}, F, Q, p, u\right) \cap w_{Z}\left(\Delta^{m}, G, Q, p, u\right) \subset w_{Z}\left(\Delta^{m}, F+G, Q, p, u\right)$;

(iv) If $q_{k}$ is stronger than $r_{k}$ for each $k \in \mathbb{N}$, then $w_{Z}\left(\Delta^{m}, F, Q, p, u\right) \subset w_{Z}\left(\Delta^{m}, F, R, p, u\right)$;

(v) If $q_{k}$ equivalent to $r_{k}$ for each $k \in \mathbb{N}$, then $w_{Z}\left(\Delta^{m}, F, Q, p, u\right)=w_{Z}\left(\Delta^{m}, F, R, p, u\right)$;

(vi) $w_{Z}\left(\Delta^{m}, F, Q, p, u\right) \cap w_{Z}\left(\Delta^{m}, F, R, p, u\right) \neq \emptyset$.

Proof. (i) We will only prove (i) for $Z=0$ and the other cases can be proved by using similar arguments. Let $\varepsilon>0$ and choose $\delta$ with $0<\delta<1$ such that $f(t)<\varepsilon$ for $0 \leq t \leq \delta$ and for all $k \in \mathbb{N}$. Write $y_{k}=g\left(q_{k}\left(u_{k} \Delta^{m} x_{k}\right)\right)$ and consider

$$
\sum_{k=1}^{n}\left[f\left(y_{k}\right)\right]=\sum_{1}\left[f\left(y_{k}\right)\right]+\sum_{2}\left[f\left(y_{k}\right)\right]
$$


where the first summation is over $y_{k} \leq \delta$ and second summation is over $y_{k}>\delta$. Since $f$ is continuous, we have

$$
\sum_{1}\left[f\left(y_{k}\right)\right]<n \varepsilon
$$

By the definition of $f$, we have for $y_{k}>\delta$,

$$
f\left(y_{k}\right)<2 f(1) \frac{y_{k}}{\delta}
$$

Hence

$$
\frac{1}{n} \sum_{2}\left[f\left(y_{k}\right)\right] \leq 2 \delta^{-1} f(1) \frac{1}{n} \sum_{k=1}^{n} y_{k}
$$

From (2.5) and (2.7), we obtain $w_{0}\left(\Delta^{m}, f, Q, u\right) \subset w_{0}\left(\Delta^{m}, f \circ g, Q, u\right)$.

The following result is a consequence of Theorem 2.5(i).

Corollary 2.6. Let $f$ be a modulus function. Then $w_{Z}\left(\Delta^{m}, Q, u\right) \subset w_{Z}\left(\Delta^{m}, f, Q, u\right)$.

Theorem 2.7. Let $0<p_{k} \leq t_{k}$ and $\left(t_{k} / p_{k}\right)$ be bounded; then $w_{Z}\left(\Delta^{m}, F, Q, t, u\right) \subset$ $w_{Z}\left(\Delta^{m}, F, Q, p, u\right)$.

Proof. If we take $w_{k}=\left[f_{k}\left(q_{k}\left(u_{k} \Delta^{m} x_{k}\right)\right)\right]^{t_{k}}$ for all $k$ and using the same technique of Theorem 5 of Maddox [19], it is easy to prove the theorem.

Theorem 2.8. Let $f$ be a modulus function; if $\lim _{t \rightarrow \infty}(f(t) / t)=\beta>0$, then $w_{1}\left(\Delta^{m}, Q, p, u\right)=$ $w_{1}\left(\Delta^{m}, f, Q, p, u\right)$.

Proof. Omitted.

\section{3. $\Delta^{m} u_{q}$-Statistical Convergence}

The notion of statistical convergence were introduced by Fast [20] and Schoenberg [21], independently. Over the years and under different names, statistical convergence has been discussed in the theory of Fourier analysis, ergodic theory, and number theory. Later on it was further investigated from the sequence space point of view and linked with summability theory by Šalát [22], Fridy [23], Connor [24], Mursaleen [25], Işik [26], Malkowsky and Savas [27], and many others. In recent years, generalizations of statistical convergence have appeared in the study of strong integral summability and the structure of ideals of bounded continuous functions on locally compact spaces. Statistical convergence and its generalizations are also connected with subsets of the Stone-Čech compactification of the natural numbers. Moreover, statistical convergence is closely related to the concept of convergence in probability. The notion depends on the density of subsets of the set $\mathbb{N}$ of natural numbers. 
A subset $E$ of $\mathbb{N}$ is said to have density positive integers which is defined by $\delta(E)$ if

$$
\delta(E)=\lim _{n \rightarrow \infty} \frac{1}{n} \sum_{k=1}^{n} X_{E}(k) \text { exists, }
$$

where $X_{E}$ is the characteristic function of $E$. It is clear that any finite subset of $\mathbb{N}$ have zero natural density and $\delta\left(E^{c}\right)=1-\delta(E)$.

In this section, we introduce $\Delta^{m} u_{q}$-statistically convergent sequences and give some inclusion relations between $\Delta^{m} u_{q}$-statistically convergent sequences and $w_{1}(f, q, p, u)$ summable sequences.

Definition 3.1. A sequence $x=\left(x_{k}\right)$ is said to be $\Delta^{m} u_{q}$-statistically convergent to $\ell$ if for every $\varepsilon>0$,

$$
\delta\left(\left\{k \in \mathbb{N}: q\left(u_{k} \Delta^{m} x_{k}-\ell\right) \geq \varepsilon\right\}\right)=0 .
$$

In this case, we write $x_{k} \rightarrow \ell\left(S_{u}^{q}\left(\Delta^{m}\right)\right)$. The set of all $\Delta^{m} u_{q}$-statistically convergent sequences is denoted by $S_{u}^{q}\left(\Delta^{m}\right)$. In the case $\ell=0$, we will write $S_{0 u}^{q}\left(\Delta^{m}\right)$ instead of $S_{u}^{q}\left(\Delta^{m}\right)$.

Theorem 3.2. Let $f$ be a modulus function; then

(i) If $x_{k} \rightarrow \ell\left(w_{1}\left(\Delta^{m}, q, u\right)\right)$, then $x_{k} \rightarrow \ell\left(S_{u}^{q}\left(\Delta^{m}\right)\right)$;

(ii) If $x \in \ell_{\infty}\left(\Delta^{m} u_{q}\right)$ and $x_{k} \rightarrow \ell\left(S_{u}^{q}\left(\Delta^{m}\right)\right)$, then $x_{k} \rightarrow \ell\left(w_{1}\left(\Delta^{m}, q, u\right)\right)$;

(iii) $S_{u}^{q}\left(\Delta^{m}\right) \cap \ell_{\infty}\left(\Delta^{m} u_{q}\right)=w_{1}\left(\Delta^{m}, q, u\right) \cap \ell_{\infty}\left(\Delta^{m} u_{q}\right)$,

where $\ell_{\infty}\left(\Delta^{m} u_{q}\right)=\left\{x \in w(X): \sup _{k} q\left(u_{k} \Delta^{m} x_{k}\right)<\infty\right\}$.

Proof. Omitted.

In the following theorems, we will assume that the sequence $p=\left(p_{k}\right)$ is bounded and $0<h=\inf _{k} p_{k} \leq p_{k} \leq \sup _{k} p_{k}=H<\infty$.

Theorem 3.3. Let $f$ be a modulus function. Then $w_{1}\left(\Delta^{m}, f, q, p, u\right) \subset S_{u}^{q}\left(\Delta^{m}\right)$.

Proof. Let $x \in w_{1}\left(\Delta^{m}, f, q, p, u\right)$ and let $\varepsilon>0$ be given. Let $\sum_{1}$ and $\sum_{2}$ denote the sums over $k \leq n$ with $q\left(u_{k} \Delta^{m} x_{k}-\ell\right) \geq \varepsilon$ and $q\left(u_{k} \Delta^{m} x_{k}-\ell\right)<\varepsilon$, respectively. Then

$$
\begin{aligned}
\frac{1}{n} \sum_{k=1}^{n}\left[f\left(q\left(u_{k} \Delta^{m} x_{k}-\ell\right)\right)\right]^{p_{k}} & =\frac{1}{n} \sum_{1}\left[f\left(q\left(u_{k} \Delta^{m} x_{k}-\ell\right)\right)\right]^{p_{k}} \\
& \geq \frac{1}{n} \sum_{1}[f(\varepsilon)]^{p_{k}} \\
& \geq \frac{1}{n} \sum_{1} \min \left([f(\varepsilon)]^{h},[f(\varepsilon)]^{H}\right) \\
& \geq \frac{1}{n}\left|\left\{k \leq n: q\left(u_{k} \Delta^{m} x_{k}-\ell\right) \geq \varepsilon\right\}\right| \min \left([f(\varepsilon)]^{h},[f(\varepsilon)]^{H}\right) .
\end{aligned}
$$

Hence $x \in S_{u}^{q}\left(\Delta^{m}\right)$. 
Theorem 3.4. Let $f$ be bounded; then $S_{u}^{q}\left(\Delta^{m}\right) \subset w_{1}\left(\Delta^{m}, f, q, p, u\right)$.

Proof. Suppose that $f$ is bounded. Let $\varepsilon>0$ and $\Sigma_{1}$ and $\Sigma_{2}$ be denoted in previous theorem. Since $f$ is bounded, there exists an integer $K$ such that $f(x)<K$, for all $x \geq 0$. Then

$$
\begin{aligned}
\frac{1}{n} \sum_{k=1}^{n}\left[f\left(q\left(u_{k} \Delta^{m} x_{k}-\ell\right)\right)\right]^{p_{k}} & \leq \frac{1}{n}\left(\sum_{1}\left[f\left(q\left(u_{k} \Delta^{m} x_{k}-\ell\right)\right)\right]^{p_{k}}+\sum_{2}\left[f\left(q\left(u_{k} \Delta^{m} x_{k}-\ell\right)\right)\right]^{p_{k}}\right) \\
& \leq \frac{1}{n} \sum_{1} \max \left(K^{h}, K^{H}\right)+\frac{1}{n} \sum_{2}[f(\varepsilon)]^{p_{k}} \\
& \leq \max \left(K^{h}, K^{H}\right) \frac{1}{n}\left|\left\{k \leq n: q\left(u_{k} \Delta^{m} x_{k}-\ell\right) \geq \varepsilon\right\}\right| \\
& +\max \left(f(\varepsilon)^{h}, f(\varepsilon)^{H}\right) .
\end{aligned}
$$

Hence $x \in w_{1}\left(\Delta^{m}, f, q, p, u\right)$.

Theorem 3.5. $S_{u}^{q}\left(\Delta^{m}\right)=w_{1}\left(\Delta^{m}, f, q, p, u\right)$ if and only if $f$ is bounded.

Proof. Let $f$ be bounded. By Theorems 3.3 and 3.4, we have $S_{u}^{q}\left(\Delta^{m}\right)=w_{1}\left(\Delta^{m}, f, q, p, u\right)$.

Conversely suppose that $f$ is unbounded. Then there exists a sequence $\left(t_{k}\right)$ of positive numbers with $f\left(t_{k}\right)=k^{2}$, for $k=1,2, \ldots$ If we choose

$$
u_{i} \Delta^{m} x_{i}= \begin{cases}t_{k}, & i=k^{2}, i=1,2, \ldots \\ 0, & \text { otherwise }\end{cases}
$$

then we have

$$
\frac{1}{n}\left|\left\{k \leq n:\left|u_{k} \Delta^{m} x_{k}\right| \geq \varepsilon\right\}\right| \leq \frac{\sqrt{n}}{n}
$$

for all $n$ and so $x \in S_{u}^{q}\left(\Delta^{m}\right)$, but $x \notin w_{1}\left(\Delta^{m}, f, q, p, u\right)$ for $X=\mathbb{C}, q(x)=|x|$ and $p_{k}=1$ for all $k \in \mathbb{N}$. This contradicts to $S_{u}^{q}\left(\Delta^{m}\right)=w_{1}\left(\Delta^{m}, f, q, p, u\right)$.

\section{References}

[1] N. R. Das and A. Choudhury, "Matrix transformation of vector valued sequence spaces," Bulletin of the Calcutta Mathematical Society, vol. 84, no. 1, pp. 47-54, 1992.

[2] M. Et, "Spaces of Cesàro difference sequences of order $r$ defined by a modulus function in a locally convex space," Taiwanese Journal of Mathematics, vol. 10, no. 4, pp. 865-879, 2006.

[3] M. Et, A. Gökhan, and H. Altinok, "On statistical convergence of vector-valued sequences associated with multiplier sequences," Ukraïns'kiŭ Matematichniu Zhurnal, vol. 58, no. 1, pp. 125-131, 2006, translation in Ukrainian Mathematical Journal, vol. 58, no. 1, pp. 139-146, 2006.

[4] I. E. Leonard, "Banach sequence spaces," Journal of Mathematical Analysis and Applications, vol. 54, no. 1, pp. 245-265, 1976. 
[5] A. Ratha and P. D. Srivastava, “On some vector valued sequence spaces $\ell_{\infty}^{(p)}\left(E_{k}, \Lambda\right)$," Ganita, vol. 47, no. 1, pp. 1-12, 1996.

[6] J. K. Srivastava and B. K. Srivastava, "Generalized sequence space $c_{0}(X, \lambda, p)$," Indian Journal of Pure and Applied Mathematics, vol. 27, no. 1, pp. 73-84, 1996.

[7] B. C. Tripathy and M. Sen, "Vector valued paranormed bounded and null sequence spaces associated with multiplier sequences," Soochow Journal of Mathematics, vol. 29, no. 3, pp. 313-326, 2003.

[8] B. C. Tripathy and S. Mahanta, "On a class of vector-valued sequences associated with multiplier sequences," Acta Mathematicae Applicatae Sinica. English Series, vol. 20, no. 3, pp. 487-494, 2004.

[9] H. Nakano, "Concave modulars," Journal of the Mathematical Society of Japan, vol. 5, pp. 29-49, 1953.

[10] I. J. Maddox, "Sequence spaces defined by a modulus," Mathematical Proceedings of the Cambridge Philosophical Society, vol. 100, no. 1, pp. 161-166, 1986.

[11] W. H. Ruckle, "FK spaces in which the sequence of coordinate vectors is bounded," Canadian Journal of Mathematics, vol. 25, pp. 973-978, 1973.

[12] T. Bilgin, "The sequence space $\ell(p, f, q, s)$ on seminormed spaces," Bulletin of the Calcutta Mathematical Society, vol. 86, no. 4, pp. 295-304, 1994.

[13] S. Pehlivan and B. Fisher, "On some sequence spaces," Indian Journal of Pure and Applied Mathematics, vol. 25, no. 10, pp. 1067-1071, 1994.

[14] A. Waszak, "On the strong convergence in some sequence spaces," Fasciculi Mathematici, no. 33, pp. 125-137, 2002.

[15] V. K. Bhardwaj, "A generalization of a sequence space of Ruckle," Bulletin of the Calcutta Mathematical Society, vol. 95, no. 5, pp. 411-420, 2003.

[16] Y. Altın, "Properties of some sets of sequences defined by a modulus function," Acta Mathematica Scientia B. English Edition, vol. 29, no. 2, pp. 427-434, 2009.

[17] H. Kizmaz, "On certain sequence spaces," Canadian Mathematical Bulletin, vol. 24, no. 2, pp. 169-176, 1981.

[18] M. Et and R. Çolak, "On some generalized difference sequence spaces," Soochow Journal of Mathematics, vol. 21, no. 4, pp. 377-386, 1995.

[19] I. J. Maddox, “On strong almost convergence," Mathematical Proceedings of the Cambridge Philosophical Society, vol. 85, no. 2, pp. 345-350, 1979.

[20] H. Fast, "Sur la convergence statistique," Colloqium Mathematicum, vol. 2, pp. 241-244, 1951.

[21] I. J. Schoenberg, "The integrability of certain functions and related summability methods," The American Mathematical Monthly, vol. 66, pp. 361-375, 1959.

[22] T. Šalát, “On statistically convergent sequences of real numbers," Mathematica Slovaca, vol. 30, no. 2, pp. 139-150, 1980.

[23] J. A. Fridy, "On statistical convergence," Analysis, vol. 5, no. 4, pp. 301-313, 1985.

[24] J. S. Connor, "The statistical and strong p-Cesàro convergence of sequences," Analysis, vol. 8, no. 1-2, pp. 47-63, 1988.

[25] M. Mursaleen, “ $\lambda$-statistical convergence," Mathematica Slovaca, vol. 50, no. 1, pp. 111-115, 2000.

[26] M. Işik, "On statistical convergence of generalized difference sequences," Soochow Journal of Mathematics, vol. 30, no. 2, pp. 197-205, 2004.

[27] E. Malkowsky and E. Savas, "Some $\lambda$-sequence spaces defined by a modulus," Archivum Mathematicum, vol. 36, no. 3, pp. 219-228, 2000. 